\title{
Un caso raro di angiomatosi epatica diffusa nell'adulto: ruolo della scintigrafia con emazie marcate nell'ambito dell'imaging diagnostico multimodale
}

The role of red blood cell scintigraphy in the multiple-modality imaging diagnosis of a rare case of diffuse hepatic hemangiomatosis in an adult

\section{Ernesto Cason $^{a, *}$, Giorgio Treglia ${ }^{b}$, Paolo Orlandi ${ }^{c}$, Giorgio Fagioli ${ }^{a}$}

\author{
${ }^{a}$ UO Medicina Nucleare, Ospedale Maggiore, Azienda USL di Bologna \\ ${ }^{\mathrm{b}}$ Istituto di Medicina Nucleare, Policlinico A. Gemelli, Università Cattolica del Sacro Cuore, Roma \\ ${ }^{\mathrm{C}}$ UO Radiologia, Ospedale Maggiore, Azienda USL di Bologna
}

Ricevuto il 3 agosto 2010; accettato il 10 gennaio 2011

disponibile online il 10 marzo 2011

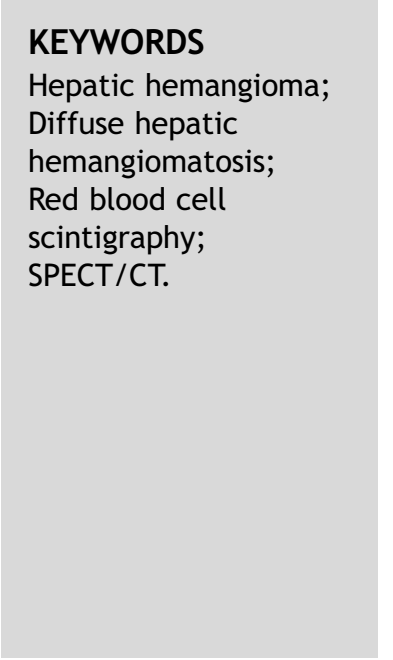

\begin{abstract}
Summary
Introduction: Angiomas are one of the most common primary tumors of the liver. Diffuse hepatic angiomatosis, however, is quite rare and usually observed in pediatric patients. We report a rare case of diffuse hepatic hemangiomatosis in a 33-year-old woman.

Case report: The patient presented with abdominal pain and a palpable upper abdominal mass. Abdominal CT and magnetic resonance imaging (MRI) findings suggested diffuse hepatic hemangiomatosis, but this finding was not confirmed by subsequent contrast-enhanced abdominal ultrasonography (US). The patient then underwent single photon emission computed tomography (SPECT)/CT scintigraphy with Tc-99m-labeling of red blood cells (RBC). This examination revealed increased uptake of labeled erythrocytes in several of the hepatic lesions corresponding to $\mathrm{CT}$ and RM findings, thereby confirming the clinical hypothesis of diffuse hepatic hemangiomatosis.

Discussion: RBC scintigraphy with SPECT/CT can facilitate the comparison of other crosssectional imaging methods such as CT and MRI. This case highlights the importance of a multiple-modality approach in the imaging diagnosis of this condition.

(c) 2010 Elsevier Srl. All rights reserved.
\end{abstract}

\footnotetext{
* Corrispondenza: UO Medicina Nucleare, Ospedale Maggiore, largo Nigrisoli 2 - 40133 Bologna.

E-mail: e.cason@ausl.bo.it (E. Cason).
} 


\section{Introduzione}

L'angioma epatico è uno dei più comuni tumori del fegato [1], mentre l'angiomatosi epatica diffusa è una patologia rara, più frequente nell'infanzia [2-7]. Riportiamo di seguito un caso di angiomatosi epatica diffusa di riscontro radiologico in un adulto, sottolineando l'importanza della scintigrafia con emazie marcate nell'ambito dell'imaging diagnostico multimodale.

Gli autori dichiarano che lo studio presentato è stato realizzato in accordo con gli standard etici stabiliti nella Dichiarazione di Helsinki, e che il consenso informato è stato ottenuto da tutti i partecipanti prima del loro arruolamento allo studio.

\section{Caso clinico}

Una donna di 33 anni con storia clinica di sarcoidosi è stata sottoposta a un esame di tomografia computerizzata (TC) dell'addome per dolore addominale persistente e massa addominale palpabile a livello dell'addome superiore all'esame obiettivo.

L'esame TC con mezzo di contrasto ha evidenziato multiple aree ipodense a livello epatico in fase venosa precoce ( fig. 1) con presa di contrasto nelle fasi tardive a livello di alcune aree, quadro compatibile con angiomatosi epatica diffusa.

La paziente è stata successivamente sottoposta a un esame di risonanza magnetica dell'addome (RM) che ha mostrato multiple lesioni epatiche ipointense rispetto al parenchima epatico nelle immagini T1 pesate e iperintense nelle immagini T2 FAT SAR pesate ( fig. $2 a$ ), con presa di contrasto di alcuni noduli in fase tardiva nelle immagini in T1 (fig. 2b), avvalorando i reperti TC. Successivamente la paziente è stata sottoposta anche a un'ecografia angiocontrastografica dell'addome che non è stata concordante con i reperti TC e RM: in particolare l'indagine, mirata allo studio

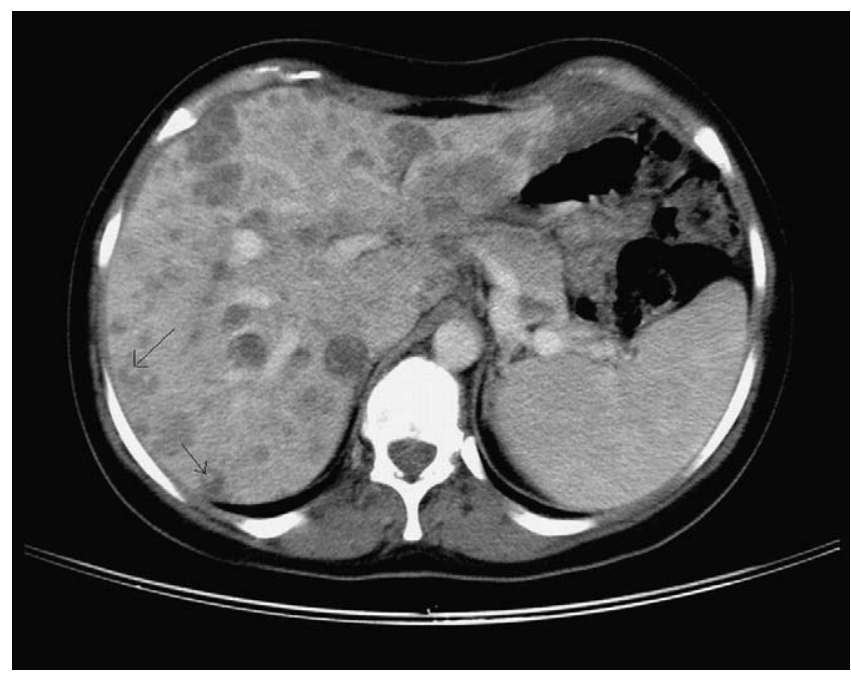

Figura 1 La TC dell'addome con mezzo di contrasto documenta multiple aree ipodense nel parenchima epatico in fase venosa.
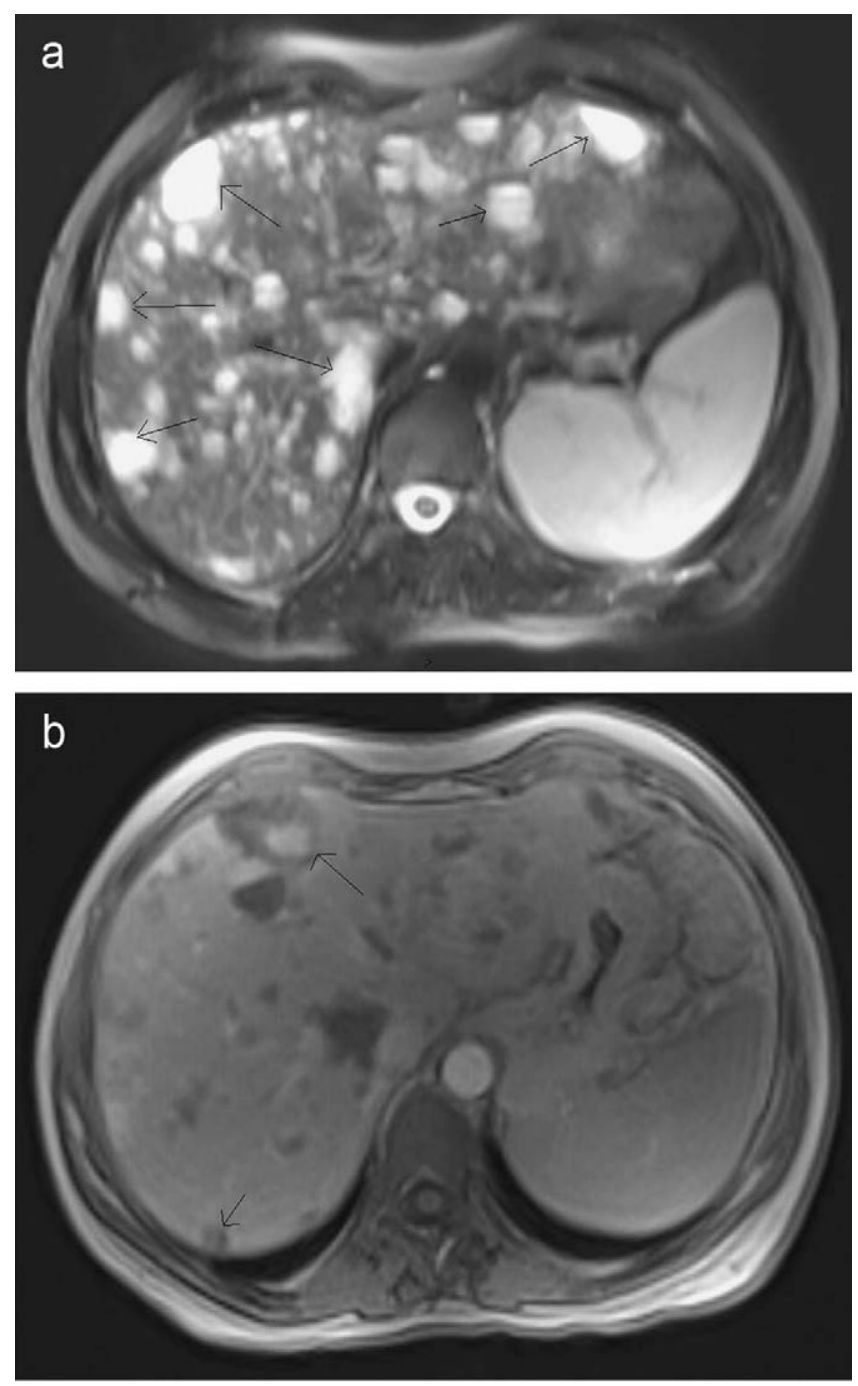

Figura 2 La RM dell'addome con mezzo di contrasto documenta multiple lesioni epatiche ipointense rispetto al parenchima epatico nelle immagini T1 pesate e iperintense nelle immagini T2 FAT SAT (a) con presa di contrasto tardiva di alcuni noduli in T1 (b), avvalorando i reperti TC di angiomatosi epatica diffusa.

della lesione di maggiore dimensioni, ha evidenziato in fase arteriosa debole vascolarizzazione periferica con alcuni spot ipervascolari contestuali, mentre in fase portale e soprattutto tardiva è stata osservata ipovascolarizzazione. Analogamente, anche le altre lesioni sono risultate ipovascolarizzate in fase tardiva. Alla luce del contesto clinico di sarcoidosi cutanea e di morbo celiaco, è stato posto sospetto ecografico per noduli epatici di granulazione o di localizzazione linfomatosa epatica.

Al fine di confermare o escludere la diagnosi di angiomatosi epatica diffusa e per poter arrivare a una decisione clinica, la paziente è stata sottoposta a una scintigrafia con emazie marcate. Dapprima, per proteggere la tiroide, sono stati somministrati alla paziente $400 \mathrm{mg}$ per os di perclorato di potassio; dopo 30 minuti, per una corretta marcatura delle emazie, sono stati somministrati $10 \mathrm{mg}$ ev di pirofosfato e infine, dopo altri 30 minuti, sono stati somministrati ev $740 \mathrm{MBq}$ di $\mathrm{Na}^{-}{ }^{99 \mathrm{~m}} \mathrm{TCO}_{4}$, in bolo [8]. Le 


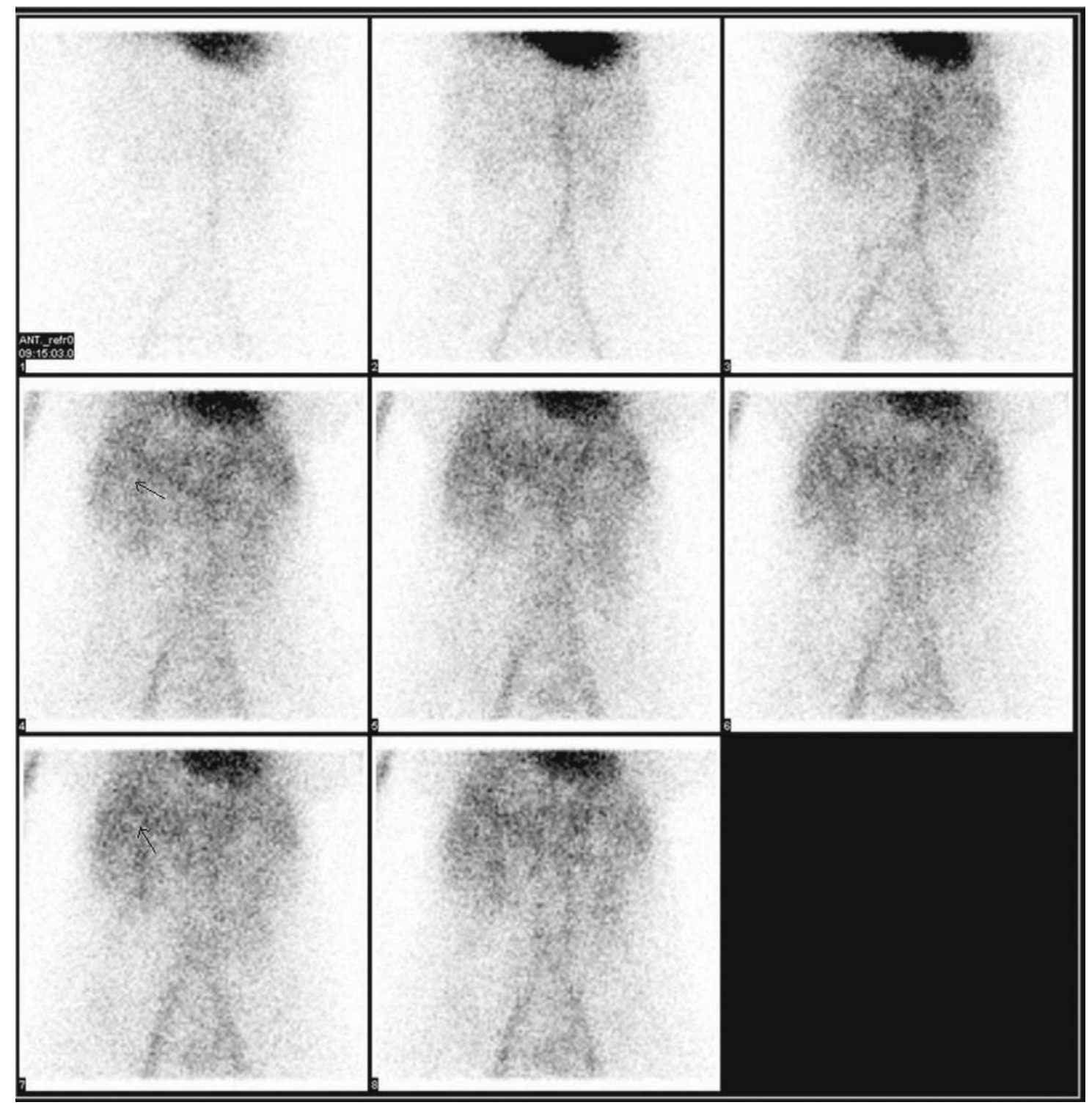

Figura 3 Scintigrafia con emazie marcate: le immagini dinamiche di perfusione (immagini precoci) documentano multiple aree di ridotta captazione del radiocomposto a livello epatico.

immagini sono state acquisite utilizzando una gammacamera ibrida SPECT/TC.

Le immagini dinamiche di perfusione sono state ottenute nei primi 2 minuti dalla somministrazione del radiocomposto e un'immagine statica precoce è stata acquisita a 5 minuti dalla somministrazione delle emazia marcate. Le immagini precoci hanno dimostrato, a livello del fegato, multiple aree di ridotta captazione del radiocomposto ( fig. 3).

Successivamente sono state acquisite anche immagini statiche tardive (a 4 ore dalla somministrazione del radiocomposto) e rilevazioni tomografiche SPECT/TC dell'addome superiore. Le immagini tardive e le rilevazioni tomografiche hanno documentato multiple aree di aumentata captazione degli eritrociti marcati in corrispondenza delle multiple lesioni di riscontro TC ( fig. 4).
Questi reperti, suggestivi per angiomatosi epatica diffusa, hanno permesso di evitare l'esecuzione di una biopsia o di un intervento chirurgico su un tessuto fortemente vascolarizzato.

\section{Discussione}

L'angioma epatico è uno dei più comuni tumori del fegato, presentandosi in oltre il 7\% della popolazione [1]. L'angiomatosi epatica diffusa è invece una condizione rara, più frequente nell'infanzia che in età adulta [2-7], la cui eziologia è sconosciuta, anche se nella sua insorgenza è stato ipotizzato un possibile ruolo degli ormoni sessuali femminili [3-9]. 


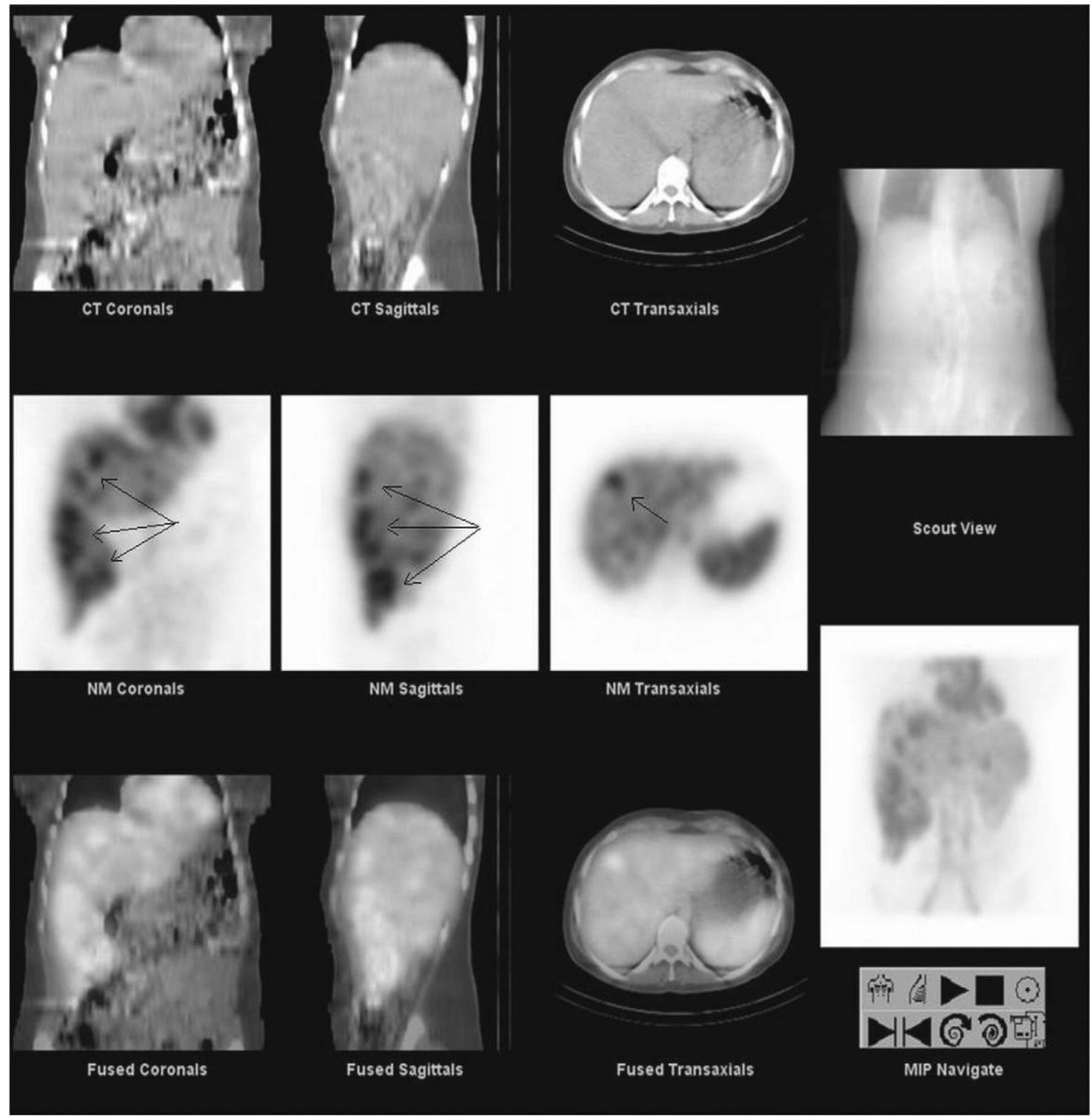

Figura 4 La rilevazione tomoscintigrafica tardiva SPECT/TC documenta multiple aree di iperaccumulo delle emazie marcate a livello epatico in corrispondenza delle aree ipodense di riscontro TC.

I pazienti con angiomatosi diffusa del fegato generalmente presentano sintomi come dolore addominale assieme al reperto obiettivo di massa palpabile a livello dell'addome superiore [2-15].

La diagnosi differenziale è rivolta soprattutto verso i tumori maligni epatici, specialmente quelli di origine vascolare, anche se al momento non vi sono sicuri reperti radiologici differenziali.

Uno o più angiomi si osservano spesso come reperto occasionale in corso di indagini TC o ecografiche [1-12]. Nelle immagini TC con mezzo di contrasto gli angiomi appaiono come aree ipodense con presa di contrasto ("contrast enhancement") nelle fasi tardive [10-12].

L'ecografia, senza e con mezzo di contrasto, può identificare la presenza di lesioni epatiche, ma non sempre riesce a chiarire con certezza la loro natura; anche la RM, benché sia comunque una metodica superiore alla TC nell'identificazione di noduli epatici, non riesce a volte a discriminare gli angiomi epatici dalle metastasi ipervascolarizzate [10-16].

Nella scintigrafia epatica con emazie marcate il reperto tipico dell'angioma a livello epatico è un'area di ipoperfusione delle emazie marcate nelle immagini di perfusione, associata a iperaccumulo focale delle emazie marcate nelle immagini tardive. Risulta preferibile aggiungere una rilevazione tomografica SPECT/TC che, combinando le informazioni funzionali SPECT con quelle anatomiche TC, permette di migliorare l'accuratezza diagnostica nel caso di localizzazioni in sedi critiche, per esempio in prossimità del cuore o vicino ai principali vasi intraepatici $[13,14]$. La scintigrafia con emazie marcate si è dimostrata una metodica sensibile e altamente specifica per la diagnosi di angiomi epatici e con un valore aggiunto nell'analisi costo-efficacia, riducendo il numero di pazienti da sottoporre a metodiche invasive come l'angiografia $[11,14]$. 
Nel caso riportato l'approccio diagnostico multimodale (inclusa la scintigrafia con emazie marcate con rilevazioni SPECT/TC) ha svolto un ruolo importante nella diagnosi di angiomatosi epatica diffusa. In accordo con i reperti scintigrafici, TC e RM, la paziente non è stata sottoposta a interventi chirurgici o altre indagini invasive, con conseguente riduzione della morbilità e dei costi.

\section{Conflitto di interessi}

Gli autori dichiarano di essere esenti da conflitto di interessi.

\section{Bibliografia}

[1] Yoon SS, Charny CK, Fong Y, Jarnagin WR, Schwartz LH, Blumgart $\mathrm{LH}$, et al. Diagnosis, management, and outcomes of 115 patients with hepatic hemangioma. J Am Coll Surg 2003;197(3): $392-402$.

[2] Jayanthi V, Shankar TR, Ravindran C, Sudalaimuthu S, Chandrasekar TS. Diffuse hepatic hemangiomatosis: case report. Trop Gastroenterol 2000;21(4):188-9.

[3] Ozakyol A, Kebapci M. Enhanced growth of hepatic hemangiomatosis in two adults after postmenopausal estrogen replacement therapy. Tohoku J Exp Med 2006;210(3):257-61.

[4] Langner C, Thonhofer R, Hegenbarth K, Trauner M. Diffuse hemangiomatosis of the liver and spleen in an adult. Pathologe 2001;22(6):424-8.

[5] Moon WS, Yu HC, Lee JM, Kang MJ. Diffuse hepatic hemangiomatosis in an adult. J Korean Med Sci 2000;15(4):471-4.

[6] Langsteger W, Lind P, Költringer P, Eber $O$. An unusual case of hepatic hemangiomatosis: sonographic, CT, and 99mTc-RBC findings. AJR Am J Roentgenol 1990;154(2):423.
[7] Kim EH, Park SY, Ihn YK, Hwang SS. Diffuse hepatic hemangiomatosis without extrahepatic involvement in an adult patient. Korean J Radiol 2008;9(6):559-62.

[8] Royal HD, Brown ML, Drum DE, Nagle CE, Sylvester JM, Ziessman HA. Procedure guideline for hepatic and splenic imaging. Society of Nuclear Medicine. J Nucl Med 1998;39(6):1114-6.

[9] Lehmann FS, Beglinger C, Schnabel K, Terracciano L. Progressive development of diffuse liver hemangiomatosis. J Hepatol 1999;30(5):951-4.

[10] Semelka RC, Martin DR, Balci C, Lance T. Focal liver lesions: comparison of dual-phase CT and multisequence multiplanar MR imaging including dynamic gadolinium enhancement. J Magn Reson Imaging 2001;13(3):397-401.

[11] Birnbaum BA, Noz ME, Chapnick J, Sanger JJ, Megibow AJ, Maguire Jr GQ, et al. Hepatic hemangiomas: diagnosis with fusion of MR, CT, and Tc-99m-labeled red blood cell SPECT images. Radiology 1991;181(2):469-74.

[12] Birnbaum BA, Weinreb JC, Megibow AJ, Sanger JJ, Lubat E, Kanamuller $\mathrm{H}$, et al. Definitive diagnosis of hepatic hemangiomas: MR imaging versus Tc-99m-labeled red blood cell SPECT. Radiology 1990;176(1):95-101.

[13] Zheng JG, Yao ZM, Shu CY, Zhang Y, Zhang X. Role of SPECT/CT in diagnosis of hepatic hemangiomas. World J Gastroenterol 2005;11(34):5336-41.

[14] Schillaci O, Danieli R, Manni C, Capoccetti F, Simonetti G. Technetium-99m-labelled red blood cell imaging in the diagnosis of hepatic haemangiomas: the role of SPECT/CT with a hybrid camera. Eur J Nucl Med Mol Imaging 2004;31(7):1011-5.

[15] Kim JD, Chang UI, Yang JM. Clinical challenges and images in GI, Diffuse hepatic hemangiomatosis involving the entire liver. Gastroenterology 2008;134(7):1830-2197.

[16] Marinis A, Kairi E, Theodosopoulos T, Kondi-Pafiti A, Smyrniotis V. Right colon and liver hemangiomatosis: a case report and a review of the literature. World J Gastroenterol 2006;12(39): 6405-7. 